\title{
Transcriptome analysis of murine thymic epithelial cells reveals age-associated changes in microRNA expression
}

\author{
ZHIBIN GUO $^{1}$, FENG CHI $^{1}$, YAN SONG ${ }^{1}$, CHANGSHAN WANG $^{1}$, RUOXING YU ${ }^{1}$, \\ TIANLI WEI ${ }^{1}$, JINGANG GUI ${ }^{2}$ and XIKE ZHU ${ }^{1}$ \\ ${ }^{1}$ Research Center, Shengjing Hospital, China Medical University, Shenyang, Liaoning 110004, P.R. China; \\ ${ }^{2}$ Department of Pharmacology and Toxicology, Geisel School of Medicine at Dartmouth, Hanover, NH 03755, USA
}

Received May 24, 2013; Accepted August 19, 2013

DOI: $10.3892 / \mathrm{ijmm} .2013 .1471$

\begin{abstract}
Age-related thymic involution is accompanied by a decrease in thymopoiesis and, thus, a deficiency in $\mathrm{T}$ cell-mediated immunity in the elderly. A number of events involved in thymic involution have been discovered; however, it remains unclear as to whether they are causes or consequences of thymic involution. These events include the degeneration of $\mathrm{T}$ cell progenitors, as well as the deterioration of the thymic stroma, which is a characteristic of thymic epithelial cell loss due to increased apoptosis and decreased cell proliferation. MicroRNAs (miRNAs) are believed to play important roles in the regulation of cell death and proliferation during the aging process. In the present study, we compared the transcriptional levels of various miRNAs in TECs from young and aged mice using microarray analysis. Quantitative PCR was performed to confirm the changes in the expression of miRNAs in the different age groups. Possible downstream targets and pathways of these miRNAs were predicted by performing bioinformatics analysis. To the best of our knowledge, this is the first study to systematically analyze the expression of miRNAs in mouse TECs and to demonstrate that miRNA expression is altered with thymic aging.
\end{abstract}

\section{Introduction}

Age-related thymic involution is manifested by a progressive reduction in the size of the thymus with profound architectural changes, the loss of thymic epithelial cells (TECs) and the replacement of the thymic stroma with adipose tissue and

Correspondence to: Professor Xike Zhu, Research Center, Shengjing Hospital, China Medical University, 36 Sanhao St., Heping District, Shenyang, Liaoning 110004, P.R. China E-mail: zhuxk@sj-hospital.org

Abbreviations: ANOVA, analysis of variance; BM, bone marrow; DN, double negative; ETPs, early T cell progenitors; PCR, polymerase chain reaction; PBS, phosphate-buffered saline; TECs, thymic epithelial cells

Key words: aging, thymic stroma, involution, microRNA peripheral lymphocytes $(1,2)$. The atrophic thymus produces a reduced number of naïve $T$ cells with a diminished diversity of T cell receptors (3). Age-related thymic involution is attributed to one of the main causes of immunosenescence: the deterioration of immunocompetence in the elderly that is characteristic of increased susceptibility to infections, insufficient response to vaccinations and an increased propensity for autoimmune diseases and cancer (4-6).

The development of $\mathrm{T}$ cells requires the importation of $\mathrm{T}$ cell progenitors to the thymus from bone marrow (BM). The early T cell progenitors (ETPs) residing in the CD4-CD8- double negative 1 (DN1) subpopulation proliferate and differentiate in response to various thymic stromal signals. Following negative and positive selection, the majority of thymocytes in the thymus undergo apoptosis. Only a very small percentage of thymocytes differentiate into mature $\mathrm{CD}^{+}{ }^{+}$or $\mathrm{CD} 8^{+}$naïve $\mathrm{T}$ cells and are exported to the periphery. Age-related thymic involution is accompanied by a reduced number and proliferation of ETPs, as well as by the marked deterioration of the thymic stroma. The change in the microenvironment of the thymus, particularly the increased apoptosis and decreased proliferation of TECs, is considered to be the determining cause for defective $\mathrm{T}$ cell development in the aged thymus (7-9).

The perturbation of gene expression levels in the thymus can influence the process of thymic involution. For example, the overexpression of the anti-apoptotic gene, myeloid cell leukemia sequence 1 (MCL1), leads to an enlarged thymus in female mice approaching advanced age (10). Targeting Ras homolog family member B (RhoB), a member of the Rho subfamily of small GTPases, in the thymic medullary epithelium results in an early thymic atrophy (11). While the trigger of thymic aging remains a mystery, the extensive pursuit of this topic has revealed that aging is associated with changes in the expression levels of several genes in thymocytes, including the E2A and LIM domain only 2 (rhombotin-like 1) (LMO2) transcriptional regulators (12). Gene array analysis of aged thymocytes has revealed age-associated changes in the mRNA levels of several genes, including those involved in oxidative phosphorylation, $\mathrm{T}$ and $\mathrm{B}$ cell receptor signaling and antigen presentation. Of note, some of the cancer-related genes and immunoglobulin genes, such as immunoglobulin M (IgM) are upregulated in 24-month-old mice. This may account for the high incidence of cancer and increased thymic B cells 
observed in aged humans (13). Alterations in the expression levels of several genes have also been found in the thymic stroma. These genes include forkhead box N1 (FoxN1), interleukin (IL)-7, keratin 8 (12-14), Wnt4 and lamina-associated polypeptide $2 \alpha$ (LAP2 $\alpha)(15)$.

MicroRNAs (miRNAs) are a family of short (average size, $22 \mathrm{bp}$ ), non-coding RNAs found in eukaryotic cells that affect gene regulation in a sequence-specific manner (16). Emerging evidence has revealed that mutations or alterations in miRNA expression correlate with the development of various types of human cancer (17), as well as other diseases (18-20). miRNAs are also involved in the regulation of the immune response, including $\mathrm{T}$ cell differentiation and sensitivity $(21,22)$. For example, miR-181a is highly expressed in the thymus and miR-155 is upregulated in mature T cells, both of which are required for $\mathrm{T}$ cell differentiation and cell-mediated immune function $(23,24)$. Knowledge of miRNA expression profiles has been gained through the detailed examination of miRNA expression at each individual developmental stage of thymocytes in the thymus by microarray and quantitative polymerase chain reaction (PCR) $(25,26)$. However, miRNA expression in the thymic stromal compartment is completely unknown. A number of studies have shown the changes in miRNA levels in various tissues in individuals of different age groups, in which miRNAs have been suggested to be novel modulators that regulate the aging process (27). Determining miRNA expression in the thymic stroma, as well as changes in expression during thymic aging would not only broaden our knowledge of $\mathrm{T}$ cell development but would also provide a deep understanding of the mechanisms underlying thymic aging.

In this study, we compared the transcriptional levels of various miRNAs in TECs from young and aged mice using microarrays. Quantitative PCR was performed to confirm the changes in the expression of miRNAs in different age groups. Possible downstream targets and pathways of these miRNAs were predicted by performing bioinformatics analysis. To the best of our knowledge, this is the first study to systematically analyze the expression of miRNAs in mouse TECs and to demonstrate that miRNA expression is altered with thymic aging.

\section{Materials and methods}

Mice and enrichment of TECs. C57BL/6 mice of different ages up to 10 months were purchased from Charles River Laboratories (Wilmington, MA, USA). Some of the 10-month-old mice were maintained in the animal facility of China Medical University, Shenyang, China until they began to age ( $\geq 19$ months). All of the mice were maintained in a specific, pathogen-free environment. All animal procedures were performed according to the protocol approved by the Experiment Animal Center of China Medical University in accordance with the recommendations in the Guide for the Care and Use of Laboratory Animals of the National Institutes of Health. The TECs were purified according to a previously described method (7). In brief, the thymic sub-capsule was gently torn apart with fine-tip forceps and shaken several times in cold phosphate-buffered saline (PBS) to remove as many thymocytes as possible. The remaining thymic tissue was further subjected to enzymatic digestion with $1 \mathrm{mg} / \mathrm{ml}$ collagenase (Invitrogen, Carlsbad, CA, USA) and $5 \mathrm{U} / \mathrm{ml}$ DNase I (Sigma-Aldrich, USA) for $10 \mathrm{~min}$ at $37^{\circ} \mathrm{C}$ with intermittent shaking. The thymic dissociated cells were stained by fluorescence-conjugated anti-CD45 (rat antibody), followed by metal beads conjugated to anti-rat IgG (Miltenyi Biotec, Bergisch Gladbach, Germany). The bead-labeled cells were subjected to LS magnetic columns to obtain TEC-enriched cells by negative selection, which were used for miRNA microarray and quantitative PCR assays.

miRNA microarray. The microarray assay was performed by the service provider Kangchen Biotech, China. Total RNA from enriched TECs of young (2-month-old) and aged (20-monthold) mice was extracted using TRIzol reagent (Invitrogen) and the RNeasy Mini kit (Qiagen, Hilden, Germany), according to the manufacturer's instructions. A total of 0.25-1 $\mu \mathrm{g}$ RNA sample was labeled Hy3 using the miRCURY ${ }^{\mathrm{TM}}$ Array Power Labeling kit (Exiqon, Aarhus, Denmark). Hybridization was performed using the miRCURY ${ }^{\mathrm{TM}}$ LNA Array (miRBase.14.0). Signal scanning was performed using the Axon GenePix 4000B microarray scanner. GenePix pro v6.0 was used to read the raw intensity of the images.

The intensity of the green signal was calculated after background subtraction and 4 replicated spots of each probe on the same slide were used to calculate the median. The median is the 50\% quantile of the miRNA intensity which is $>50$ in all samples after background correction. The median normalization method was used to obtain normalized data. Clustering and statistical analysis were performed on the normalized data of miRNAs.

Quantitative PCR. Total RNA (including small RNA) was extracted from the TECs of young (1-month-old), young adults (6-month-old), middle-aged (10-month-old) and aged (19-month-old) mice using the RNeasy Mini kit (Qiagen). A poly(A) tail was added to 3' end of small RNA [E. coli Poly(A) Polymerase; New England Biolabs (NEB), Ipswich, MA, USA] before the first-strand cDNA was synthesized using the RT Reagent kit (Takara, Otsu, Japan). RNA with no Poly(A) Polymerase was used as the internal control. Instead of using a common oligo(dT) primer or a random primer, a specific oligo(dT) primer with the following sequence was used: 5'-GCTGTCAACGATA CGCTACGTAACGGCATGACAGT G(T)24V-3'. cDNA $(1 \mu \mathrm{l})$ was used as the PCR template. The results were normalized to U6 snRNA that was used as the internal reference (Ambion Inc., Foster City, CA, USA). The forward primers for miRNAs are listed in Table I and every PCR reaction for miRNA quantification shared the same reverse primer as follows: 5'-GCTGTCAACGATACGCTA CGTAACG-3'.

Quantitative PCR was performed using the 7500 Real Time PCR System (Applied Biosystems, Foster City, CA, USA) and the SYBR-Green PCR master mix (Takara) with 40 cycles of $95^{\circ} \mathrm{C}$ for $15 \mathrm{sec}$ and $62^{\circ} \mathrm{C}$ for $90 \mathrm{sec}$. Specificity of amplification was confirmed by melting curve analyses.

Bioinformatics prediction of the targets of miRNAs. The targets of the miRNAs were predicted using the TargetScan, PicTar, miRanda and EIMMo databases. The predicted hits from each algorithm were sorted as per the scores. The congruent high score hits in all 4 of the algorithms were considered to be reliable predictions. 
Table I. Primers of the 20 miRNAs used in qPCR.

\begin{tabular}{ll}
\hline miRNA & \multicolumn{1}{c}{ Primer sequence } \\
\hline miR-146a & tgagaactgaattccatgggtt \\
miR-148b & tcagtgcatcacagaacttgta \\
miR-150 & ccaaccttgtaccagtgaaa \\
miR-154-3p & cgaatcatacacggttgacctatt \\
miR-155 & ttaatgctaattgtgatagggg \\
miR-181a & cattcaacgctgtcggtgagt \\
miR-181b & attcattgctgtcggtgggaa \\
miR-181c & aacattcaacctgtcggtgagt \\
miR-192 & gctgacctatgaattgacagcc \\
miR-194 & tgtaacagcaactccatgtgga \\
miR-19a & tgtgcaaatctatgcaaaactga \\
miR-19b & gcaaatccatgcaaaactga \\
miR-22 & aagctgccagttgaagaactgt \\
miR-24 & tggctcagttcagcagga \\
miR-322 & gcagcaattcatgttttgga \\
miR-382-3p & ctctgtcattcacggacaaca \\
miR-431 & caggccgtcatgcaaaa \\
miR-465a-3p & gatcagggcctttctaagta \\
miR-93 & aagtgctgttcgtgcaggtag \\
miR-96 & tggcactagcacattttgct \\
\hline
\end{tabular}

qPCR, quantitative polymerase chain reaction; miRNA, microRNA.

Statistical analysis. A t-test was used for comparing the miRNA expression levels in the 2- and 20-month-old groups. One-way analysis of variance (ANOVA) was used for comparing the PCR results or the weight of the thymus between the 1-, 6-, 10and 19-month-old groups. A linear correlation test was used for comparing miRNA expression and changes in thymic weight change during aging. A probability value ( $p$-value) $<0.05$ was considered to indicate a statistically significant difference. Each statistic and derived figure was prepared using GraphPad Prism-5 software.

\section{Results}

Microarray data reveal the difference in the expression levels of miRNAs in TECs from young and aged thymus. To determine the age-related changes in miRNA expression in thymic stromal tissue, we compared miRNA expression in enriched TECs from the thymi of 2- and 20-month-old mice by miRNA microarray analysis. Hierarchical clustering analysis partitioned the samples into 2 groups, 2-month-old (young; sample 1 and 2) and 20-month-old (aged; sample 3 and 4), which suggested that each sample had a distinct miRNA expression profile (Fig. 1A). Among the 678 mouse miRNA probes in the miRNA arrays, $111(16.4 \%)$ miRNAs were expressed and detected in the TECs from the 2 groups (fold change $>2$ or $<0.5)$. From the data analysis based on the p-values (t-test), 31 miRNAs were upregulated and 22 were
Table II. Expression pattern of 20 miRNAs as shown by microarray analysis.

\begin{tabular}{|c|c|c|c|c|}
\hline \multirow[b]{2}{*}{ miRNA } & \multicolumn{2}{|c|}{ Normalization } & \multirow[b]{2}{*}{ Fold $^{\mathrm{a}}$} & \multirow[b]{2}{*}{$\mathrm{p}$-value } \\
\hline & $\begin{array}{l}\text { 2-month- } \\
\text { old mice }\end{array}$ & $\begin{array}{l}\text { 20-month- } \\
\text { old mice }\end{array}$ & & \\
\hline miR-382-3p & 0.0165 & 0.1139 & 6.8919 & 0.0067 \\
\hline miR-154-3p & 0.0166 & 0.0887 & 5.3488 & 0.0175 \\
\hline $\operatorname{miR}-431$ & 0.0377 & 0.1847 & 4.8985 & 0.0361 \\
\hline miR-465a-3p & 0.0361 & 0.1522 & 4.2228 & 0.0133 \\
\hline miR-146a & 0.1065 & 0.0192 & 0.1805 & 0.0343 \\
\hline miR-181c & 0.3194 & 0.1035 & 0.3241 & 0.0338 \\
\hline miR-322 & 0.7906 & 0.2762 & 0.3494 & 0.0136 \\
\hline miR-194 & 0.8985 & 0.3531 & 0.3930 & 0.0150 \\
\hline miR-19b & 9.8918 & 4.2293 & 0.4276 & 0.0142 \\
\hline miR-19a & 11.2469 & 4.8793 & 0.4338 & 0.0378 \\
\hline miR-181a & 0.6530 & 0.2839 & 0.4348 & 0.0195 \\
\hline miR-96 & 0.9457 & 0.4177 & 0.4417 & 0.0064 \\
\hline miR-155 & 2.2002 & 0.9730 & 0.4422 & 0.0059 \\
\hline miR-24 & 10.5154 & 4.7114 & 0.4480 & 0.0422 \\
\hline miR-181b & 0.2506 & 0.1127 & 0.4496 & 0.0120 \\
\hline $\mathrm{miR}-148 \mathrm{~b}$ & 0.1819 & 0.0845 & 0.4646 & 0.0260 \\
\hline miR-150 & 1.9059 & 0.8968 & 0.4705 & 0.0324 \\
\hline miR-192 & 0.3752 & 0.1773 & 0.4725 & 0.0184 \\
\hline miR-22 & 3.8834 & 1.8492 & 0.4762 & 0.0213 \\
\hline miR-93 & 1.0102 & 0.4813 & 0.4764 & 0.0151 \\
\hline
\end{tabular}

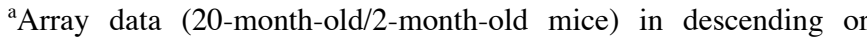
ascending order. ${ }^{\mathrm{b}} \mathrm{p}<0.05$, statistically significant as shown by t-test. miRNA, microRNA.

downregulated significantly $(\mathrm{p}<0.05)$ in the TECs from the aged thymus (Fig. 1B). The 20 miRNAs that had a greater fold change in expression than the other 33 were selected for quantification by quantitative PCR (Fig. 1C). Among the 20 miRNAs that were analyzed, 4 (miR-382-3p, miR-154-3p, miR-431 and miR-465a-3p) were upregulated in the aged TECs, whereas the other 16 miRNAs were downregulated (Table II). The upregulated miRNAs as measured in the microarray assay increased by at least 4-fold and the downregulated miRNAs were decreased by at least 0.5 -fold in the aged TECs compared with those from the 2-month-old (young) mice.

Quantitative PCR confirms the difference in miRNA expression in young and aged TECs. To confirm our results obtained from the microarray assay, we performed a more thorough investigation of miRNA expression in mouse TECs by quantitative PCR, a method with better sensitivity than microarray analysis. To be able to observe the trend in the changes in miRNA expression with thymic aging, we compared the miRNA expression levels in TECs from mice of various ages, including 1-month-old (young), 6-month-old (young adults), 10-month-old (middle-aged) and 19-month-old (aged) mice. 
A

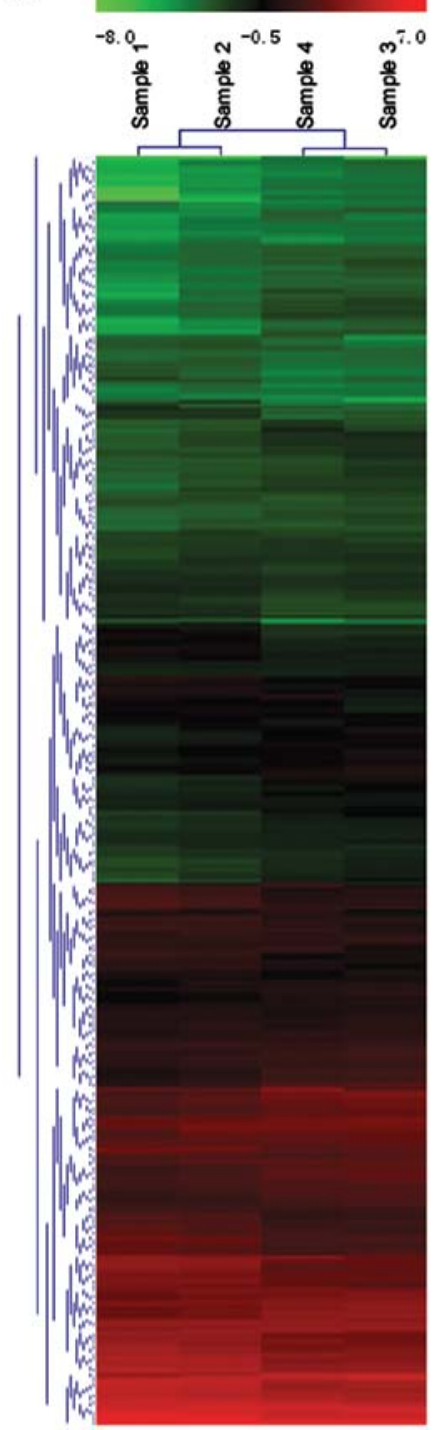

B

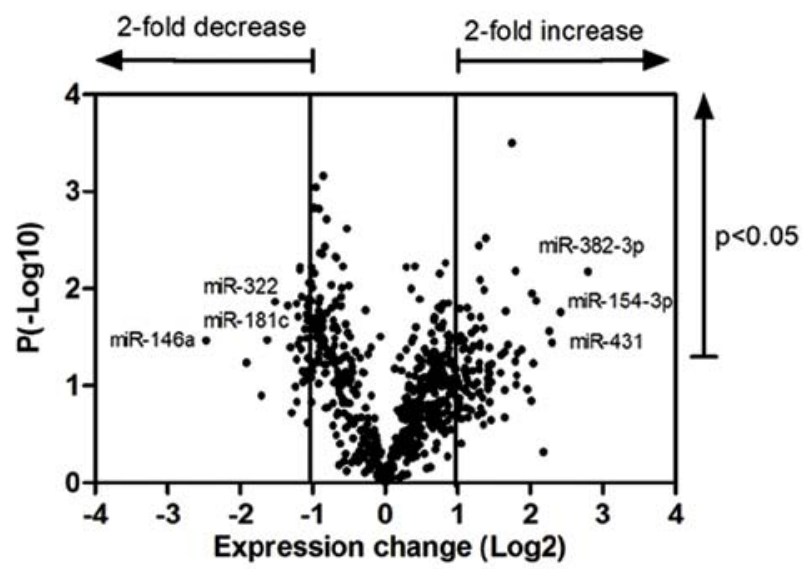

$\mathrm{C}$

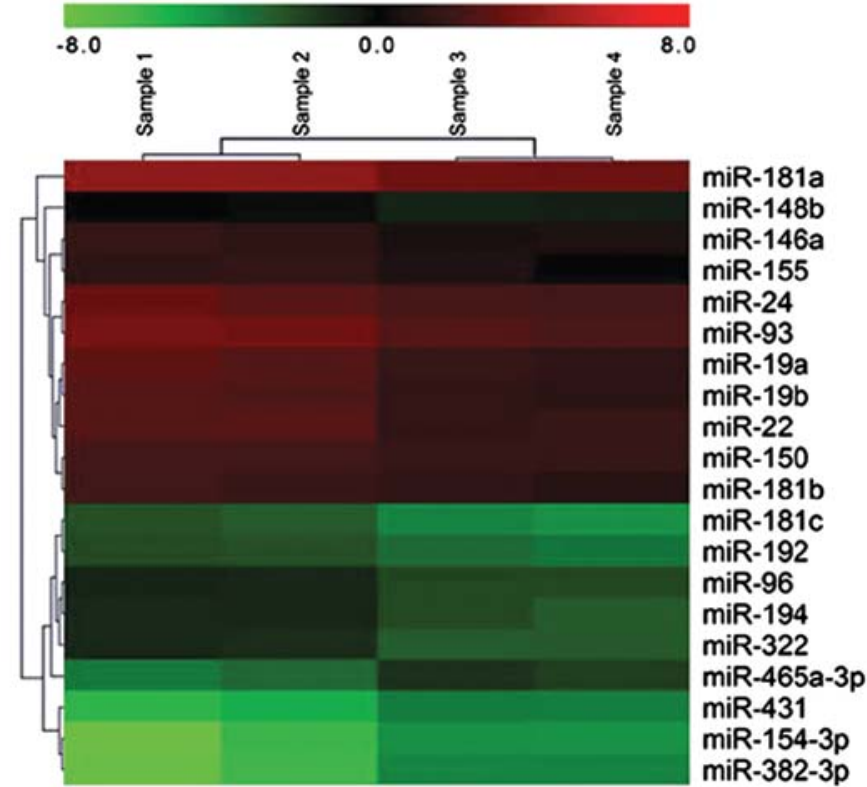

Figure 1. Results of microarray analysis of microRNA (miRNA) expression in young and aged mouse thymic epithelial cells (TECs). (A) The miRNA clustering tree of young and age mouse TECs. (B) Analysis of microarray data. Graph shows -Log10 p-values with Log2 fold changes calculated from spot replicate medians. Names of miRNAs in further quantitative reverse-transcription polymerase chain reaction validation are displayed. (C) Cluster analysis of the expression of 20 miRNAs.

The results of quantitative PCR were in agreement with the majority of the results obtained from microarray analysis. The expression of 17 out of 20 miRNAs in the 19-month-old group decreased significantly, unlike that in the 1-month-old group (all 15 miRNAs in Fig. 2 and miR-382-3p and miR-431 in Fig. 3). The decrease in the expression levels of miR-382-3p and miR431 based on quantitative PCR (Fig. 3) is not consistent with the results obtained from microarray analysis, as the expression of these 2 miRNAs was shown to be upregulated by microarray analysis (Table II). The expression of miR-154-3p and miR-465a-3p, which was also upregulated in the aged mice as shown by the results of microarray analysis, displayed no change in the different age-group samples as shown by the results of quantitative PCR (Fig. 3). The inconsistency between microarray and quantitative PCR may be the result of the variation in miRNA expression in each individual mouse. By retrospective examination of the microarray data, we found that the absolute values of the fluorescence intensity of these 4 upregulated miRNAs (miR-382-3p, miR-431, miR-154-3p and $\mathrm{miR}-465 \mathrm{a}-3 \mathrm{p}$ ) belong to the lower end of the detection limit in the microarray. In other words, the microarray data for these miRNAs may be less reliable than that obtained by quantitative PCR (Table II). In addition, even though miR-148b expression showed an age-associated decrease by quantitative PCR, which conforms to the trend observed in the microarray data, the difference in the expression in each age group was not significant (Fig. 3).

Changes in miRNA expression in TECs with age closely correlate with age-related thymic atrophy. Thymic weight loss is a reliable index of thymic aging and diminished thymopoiesis. By measuring the weight of the thymus of mice with different ages ranging from 1 to 19 months, we found that the thymus loses its weight in a biphasic pattern. 

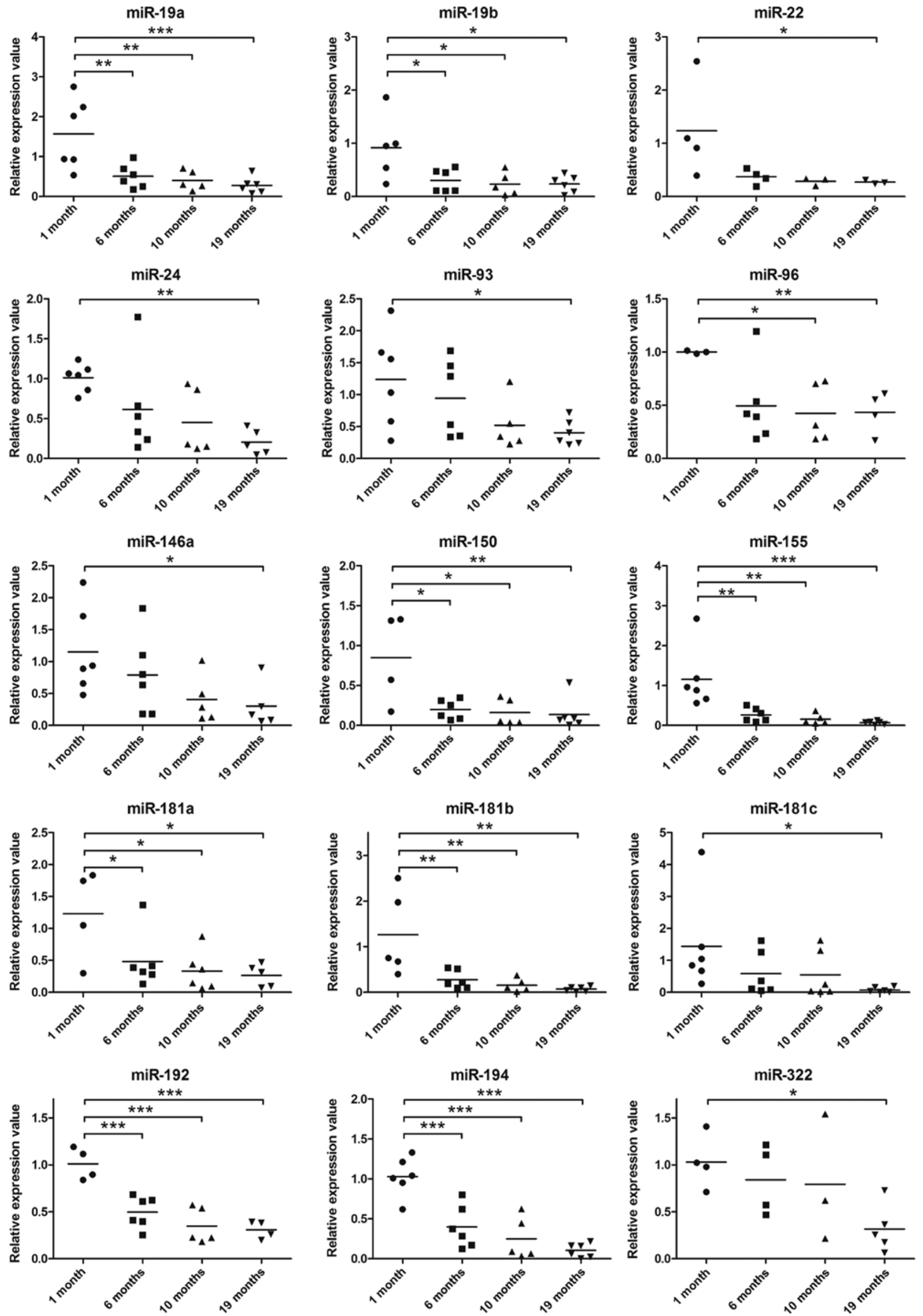

Figure 2. Quantitative polymerase chain reaction identification results of the expression of 15 microRNAs that were consistent with the results from microarray analysis. $\mathrm{n}=3-6,{ }^{*} \mathrm{p}<0.05 ;{ }^{* *} \mathrm{p}<0.01 ;{ }^{* * *} \mathrm{p}<0.001$. 

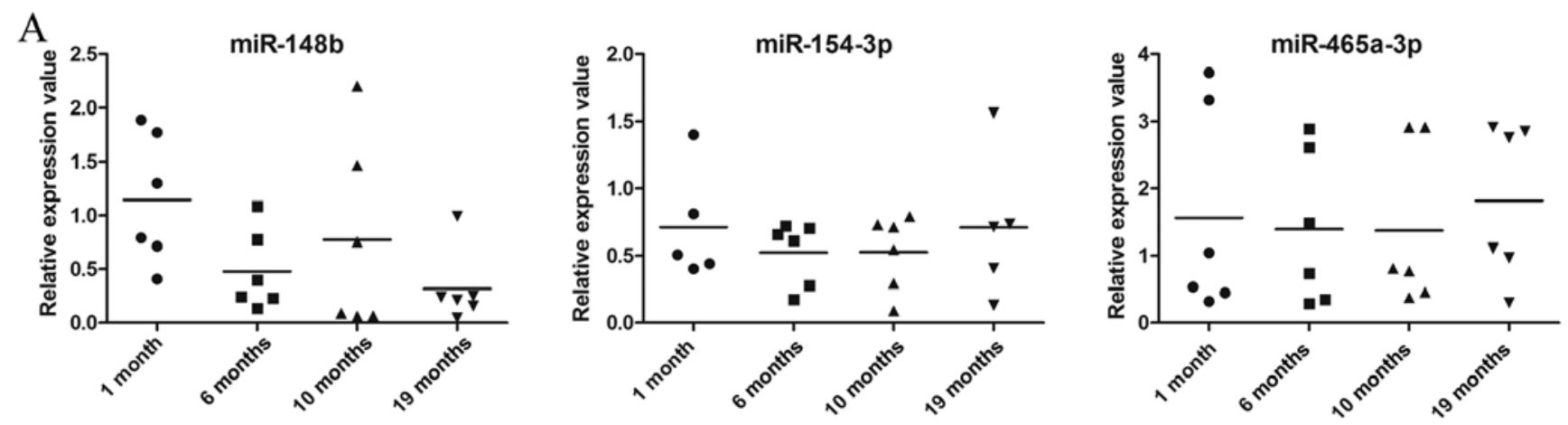

B

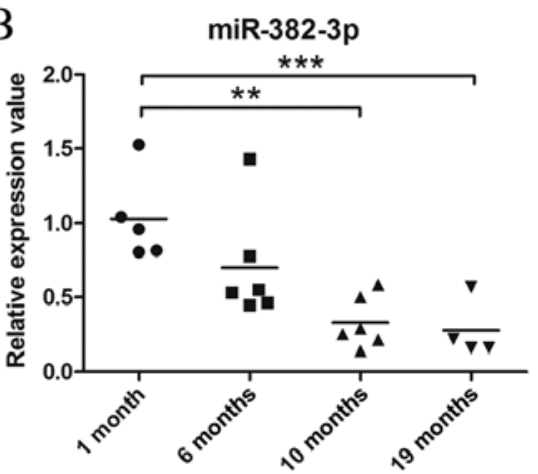

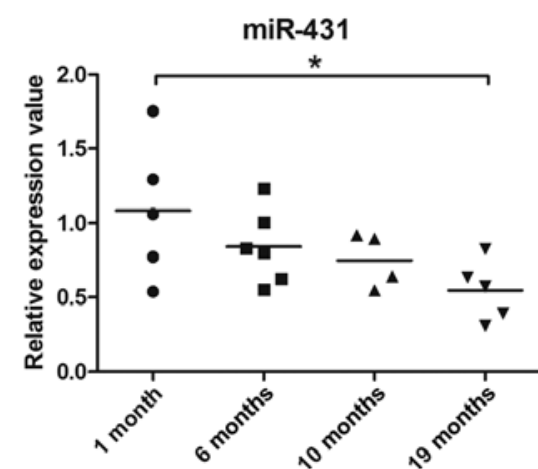

Figure 3. Quantitative polymerase chain reaction (qPCR) identification results of the expression of 5 microRNAs (miRNAs) that were not consistent with the results from microarray analysis. (A) The miRNA expression levels of miR-148b, miR-154-3p and miR-465a-3p did not differ between the groups. (B) The miRNA expression levels of miR-382-3p and miR-431 that were shown to be downregulated by qPCR but upregulated by microarray analysis during aging. $\mathrm{n}=4-6,{ }^{*} \mathrm{p}<0.05 ;{ }^{* *} \mathrm{p}<0.01 ;{ }^{* * *} \mathrm{p}<0.001$.

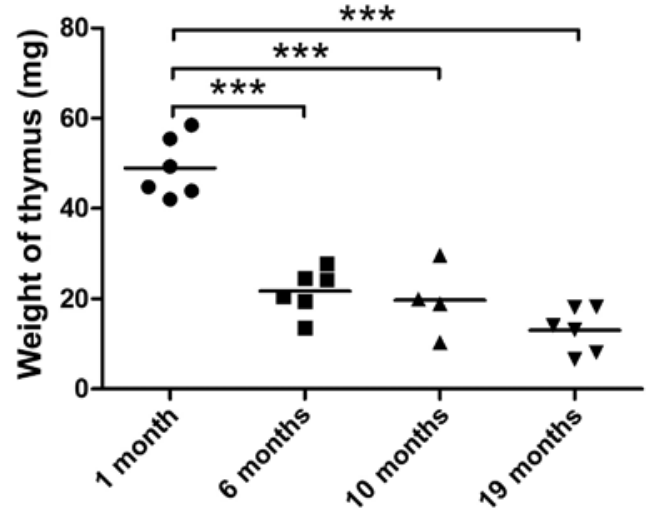

Figure 4. Marked changes in thymic weight by aging. $\mathrm{n}=4-6,{ }^{* * * *} \mathrm{p}<0.001$.

Thymic weight markedly decreased from 1 to 6 months and it decreased at a much slower rate from 6 to 19 months (Fig. 4). Of note, the expression of 13 miRNAs in the TECs (miR-194, miR-192, miR-155, miR-150, miR-19a, miR-19b, miR-148b, miR-382-3p, miR-146a, miR-93, miR-181a, miR-181b and miR-181c), as measured by quantitative PCR, rapidly decreased from 1 to 6 months and was only slightly altered from 6 to 10 and 19 months (Figs. 2 and 3), which is reminiscent of the pattern of thumic weight loss with age. These 13 miRNAs are possibly closely involved in the process of age-related thymic involution, whereas age-associated changes in the expression of the other 7 miRNAs did not follow the biphasic pattern of thymic weight loss (Table III). miR-465a-3p and miR-154-3p are the only 2 miRNAs in the TECs that showed an increasing trend in expression with age; however, this was not statistically significant.

Possible targets of miRNAs predicted possible pathways involved in age-related thymic involution. miRNAs exert their functions through multiple downstream targets, including a number of transcription factors, apoptosis control factors and other important factors for cell function. In the present study, we predicted the targets of these miRNAs by comparing the targets from 4 databases: Target Scan, PicTar, Miranda and EIMMo. These miRNAs not only differed in expression between the young and aged TECs (Fig. 2 and miR-382-3p and miR-431 in Fig. 3), but also closely correlated with changes in thymic weight with aging (Table III). We paid much attention to the predictions of targets implicated to play a role in thymic aging. We selected the output hits with a high score in all 4 databases for each of the analyzed miRNAs. The predicted targets are listed in Table IV.

\section{Discussion}

The thymus is responsible for thymocyte development and de novo $\mathrm{T}$ cell production. Age-related thymic involution is one of the prominent causes of $\mathrm{T}$ cell-mediated immunodeficiency in the elderly. Emerging evidence indicates that the deterioration of the thymic microenvironment, particularly the loss of 
Table III. Linear correlation analysis of the expression of 20 miRNAs (by qPCR) and changes in thymic weight with aging.

\begin{tabular}{lll}
\hline Name & R-value & p-value \\
\hline miR-155 & 0.6957 & $0.0002^{\mathrm{b}}$ \\
miR-181b & 0.632 & $0.0012^{\mathrm{b}}$ \\
miR-194 & 0.6077 & $0.0016^{\mathrm{b}}$ \\
miR-19b & 0.5993 & $0.002^{\mathrm{b}}$ \\
miR-181c & 0.5886 & $0.0025^{\mathrm{b}}$ \\
miR-150 & 0.5733 & $0.0053^{\mathrm{b}}$ \\
miR-19a & 0.5477 & $0.0056^{\mathrm{b}}$ \\
miR-181a & 0.5328 & $0.0129^{\mathrm{b}}$ \\
miR-192 & 0.5155 & $0.0168^{\mathrm{b}}$ \\
miR-148b & 0.4602 & $0.0236^{\mathrm{b}}$ \\
miR-382-3p & 0.4555 & $0.038^{\mathrm{b}}$ \\
miR-146a & 0.4505 & $0.0354^{\mathrm{b}}$ \\
miR-93 & 0.4168 & $0.0428^{\mathrm{b}}$ \\
miR-322 & 0.4428 & $0.0859^{\mathrm{c}}$ \\
miR-96 & 0.4193 & $0.0833^{\mathrm{c}}$ \\
miR-22 & 0.3682 & $0.1102^{\mathrm{c}}$ \\
miR-24 & 0.2783 & $0.188^{\mathrm{c}}$ \\
miR-431 & 0.2597 & $0.2688^{\mathrm{c}}$ \\
miR-465a-3p & -0.1506 & $0.4824^{\mathrm{c}}$ \\
miR-154-3p & -0.2017 & $0.4376^{\mathrm{c}}$ \\
\hline
\end{tabular}

${ }^{\mathrm{a}} \mathrm{R}$, correlation coefficient. The higher the R-value, the stronger the correlation. ${ }^{b}$ Statistically significant correlation. ${ }^{c}$ Statistically insignificant correlation. miRNA, microRNA; qPCR, quantitative polymerase chain reaction.

TECs, largely contributes to the defect in T cell development in the thymus (7-9). Changes in several critical genes associated with age-related thymic involution have been extensively investigated $(12,15)$. To our knowledge, this is the first study to explore miRNA expression in TECs and the correlation between changes in miRNA expression with age-related thymic weight loss. By microarray and quantitative PCR analyses, our findings indicated that the majority of the 20 miRNAs that we examined had decreased expression levels with thymic aging. These results are in agreement with those from a study using peripheral blood mononuclear cells, in which >800 miRNAs were profiled and the majority of the studied miRNAs decreased in abundance with age (28). In this study, the fluorescence intensity of $\mathrm{miR}-431, \mathrm{miR}-154-3 \mathrm{p}, \mathrm{miR}-382-3 \mathrm{p}$ and miR-465a-3p, as shown by the microarray analysis was very weak, indicating that the expression of these miRNAs in the TECs of both young and aged mice was very low. Given the better sensitivity and larger sample size that was used in quantitative PCR compared with the microarray analysis, we have more confidence in the results from quantitative PCR for these 4 miRNAs than in the results from the microarray analysis.

It should be noted that several of the miRNAs that we analyzed have been implicated in important immune functions. A number of studies have confirmed that miR-155,
Table IV. Predicted target genes of 12 miRNAs found in 4 databases (TargetScan, PicTar, Miranda and EIMMo).

\begin{tabular}{ll}
\hline miRNA & \multicolumn{1}{c}{ Target genes } \\
\hline miR-155 & Myb, Hivep2, Ptpn2 \\
miR-181b & Rnf34 \\
miR-194 & Ptpn12, Hbegf, Sumo2 \\
miR-19b & Rin2, Nbea, Dlx1 \\
miR-181c & Zfp14 \\
miR-150 & Myb \\
miR-19a & Zmynd11, Rnf11, Phtf2 \\
miR-181a & Klf6, Fbxo33, Zfp3612 \\
miR-192 & Wnk1, Msn, Cdc6 \\
miR-146a & Irak1 \\
miR-93 & Epha4, Zfp367, Rab5b \\
miR-382-3p & none \\
\hline
\end{tabular}

miRNA, microRNA.

miR-181a and miRNAs derived from the miR-17-92 cluster (miR-19a and miR-19b in the present study) are involved in the development and differentiation of lymphoid and myeloid cells $(25,29-31)$. To the best of our knowledge, this is the first study to provide evidence that these miRNAs with important immunological functions are expressed in TECs.

Another important finding in this study comes from the correlation analysis between changes in the expression of miRNAs with age and the age-related thymic weight loss. In total, 13 out of the 20 miRNAs showed a significant correlation. The expression of all 13 miRNAs showed a positive correlation with the thymic weight loss during aging. It is very likely that the decrease in miRNA expression in the TECs precedes the age-induced thymic involution. This may be particularly true for miRNAs, such as miR-194, miR-192, miR-155, miR-19a, miR-19b, miR-181a and miR-181b from the 13 miRNAs, as the decrease in the expression of these miRNAs with age follows a more obvious biphasic pattern [1 to 6 months compared with 6 months onwards (Fig. 2)], which conforms to the pattern of thymic weight loss during aging (Fig. 4).

We predicted 3 target genes for miRNAs that may be involved in the process of age-related thymic involution (Table IV). Some of the targets have previously been implicated in immune functions, as well as T cell functions. For example, Myb regulates T cell differentiation (32) and the development of hematopoietic stem cells (33). Hivep2, a target gene of miR-155, plays a critical role in signal transduction $(34,35)$, lymphocyte development (36) and the production of memory $\mathrm{T}$ helper cells $(37,38)$. Wnk1 is required for mitosis $(39,40)$, proliferation and migration (41) and modulates of TGF $\beta$-Smad pathways (42). The IRAK family of genes may be involved in the expression of inflammatory gene (43). One of these genes, Irak1 may disrupt the balance of the generation of proinflammatory cytokines and type I interferons in the inherent immune response (44) and has been identified as a danger gene in systemic lupus erythematosus (45). 
Based on the targets of miRNAs, our belief is that further analysis of these targets may aid in the understanding of how these miRNAs control the thymic aging process. It can be concluded that changes in the expression of these miRNAs in TECs may at least partially regulate thymocyte development and $\mathrm{T}$ cell maturation, considering the important role that TECs play in the thymic microenvironment for thymocyte development and differentiation.

\section{Acknowledgements}

The present study was supported by the National Natural Scientific Foundation of China (grant no. 30872715, 81270430), by the Special Research Fund for Doctoral Program of Education Department of China (grant no. 20112104110011) and by the Free Research Program Fund of Shengjing Hospital (grant no. 200805) to X.Z.

\section{References}

1. Aw D, Silva AB, Maddick M, et al: Architectural changes in the thymus of aging mice. Aging Cell 7: 158-167, 2008.

2. Aw D and Palmer DB: The origin and implication of thymic involution. Aging Dis 2: 437-443, 2011.

3. Naylor K, Li G, Vallejo A N, et al: The influence of age on T cell generation and TCR diversity. J Immunol 174: 7446-7452, 2005.

4. Maue AC, Yager EJ, Swain SL, et al: T-cell immunosenescence: lessons learned from mouse models of aging. Trends Immunol 30: 301-305, 2009

5. Dorshkind K and Swain S: Age-associated declines in immune system development and function: causes, consequences, and reversal. Curr Opin Immunol 21: 404-407, 2009.

6. Foster AD, Sivarapatna A and Gress RE: The aging immune system and its relationship with cancer. Aging health 7: 707-718, 2011.

7. Gui J, Zhu X, Dohkan J, et al: The aged thymus shows normal recruitment of lymphohematopoietic progenitors but has defects in thymic epithelial cells. Int Immunol 19: 1201-1211, 2007.

8. Zhu X, Gui J, Dohkan J, et al: Lymphohematopoietic progenitors do not have a synchronized defect with age-related thymic involution. Aging Cell 6: 663-672, 2007.

9. Gui J, Mustachio LM, Su DM and Craig RW: Thymus size and age-related thymic involution: early programming, sexual dimorphism, progenitors and stroma. Aging Dis 3: 280-290, 2012.

10. Gui J, Morales AJ, Maxey SE, et al: MCL1 increases primitive thymocyte viability in female mice and promotes thymic expansion into adulthood. Int Immunol 23: 647-659, 2011.

11. Bravo-Nuevo A, O'Donnell R, Rosendahl A, et al: RhoB deficiency in thymic medullary epithelium leads to early thymic atrophy. Int Immunol 23: 593-600, 2011.

12. Ortman CL, Dittmar KA, Witte PL and Le PT: Molecular characterization of the mouse involuted thymus: aberrations in expression of transcription regulators in thymocyte and epithelial compartments. Int Immunol 14: 813-822, 2002.

13. Lustig A, Carter A, Bertak D, et al: Transcriptome analysis of murine thymocytes reveals age-associated changes in thymic gene expression. Int J Med Sci 6: 51-64, 2009.

14. Sun L, Guo J, Brown R, et al: Declining expression of a single epithelial cell-autonomous gene accelerates age-related thymic involution. Aging Cell 9: 347-357, 2010.

15. Kvell K, Varecza Z, Bartis D, et al: Wnt4 and LAP2alpha as pacemakers of thymic epithelial senescence. PLoS One 5: e10701, 2010.

16. Weatherall DJ: Thalassaemia: the long road from bedside to genome. Nat Rev Genet 5: 625-631, 2004.

17. Esquela-Kerscher A and Slack FJ: Oncomirs - microRNAs with a role in cancer. Nat Rev Cancer 6: 259-269, 2006.

18. Zhao Y, Ransom JF, Li A, et al: Dysregulation of cardiogenesis, cardiac conduction, and cell cycle in mice lacking miRNA-1-2. Cell 129: 303-317, 2007.

19. Mencia A, Modamio-Høybjør S, Redshaw N, et al: Mutations in the seed region of human miR-96 are responsible for nonsyndromic progressive hearing loss. Nat Genet 41: 609-613, 2009.
20. Feng J, Sun G, Yan J, et al: Evidence for X-chromosomal schizophrenia associated with microRNA alterations. PLoS One 4: e6121, 2009.

21. Muljo SA, Ansel KM, Kanellopoulou C, et al: Aberrant T cell differentiation in the absence of Dicer. J Exp Med 202: 261-269, 2005.

22. Li QJ, Chau J, Ebert PJ, et al: miR-181a is an intrinsic modulator of T cell sensitivity and selection. Cell 129: 147-161, 2007.

23. Chen CZ, Li L, Lodish HF and Bartel DP: MicroRNAs modulate hematopoietic lineage differentiation. Science 303: 83-86, 2004.

24. Rodriguez A, Vigorito E, Clare S, et al: Requirement of bic/microRNA-155 for normal immune function. Science 316: 608-611, 2007.

25. Neilson JR, Zheng GX, Burge CB and Sharp PA: Dynamic regulation of miRNA expression in ordered stages of cellular development. Genes Dev 21: 578-589, 2007.

26. Virts EL and Thoman ML: Age-associated changes in miRNA expression profiles in thymopoiesis. Mech Ageing Dev 131: 743-748, 2010.

27. Chen LH, Chiou GY, Chen YW, et al: MicroRNA and aging: a novel modulator in regulating the aging network. Ageing Res Rev 9 (Suppl 1): S59-S66, 2010.

28. Noren Hooten N, Abdelmohsen K, Gorospe M, et al: microRNA expression patterns reveal differential expression of target genes with age. PLoS One 5: e10724, 2010

29. O'Connell RM, Rao DS, Chaudhuri AA, et al: Sustained expression of microRNA-155 in hematopoietic stem cells causes a myeloproliferative disorder. J Exp Med 205: 585-594, 2008.

30. Ventura A, Young AG, Winslow MM, et al: Targeted deletion reveals essential and overlapping functions of the miR-17 through 92 family of miRNA clusters. Cell 132: 875-886, 2008.

31. Xiao C, Srinivasan L, Calado DP, et al: Lymphoproliferative disease and autoimmunity in mice with increased miR-17-92 expression in lymphocytes. Nat Immunol 9: 405-414, 2008.

32. Sheiness D and Gardinier M: Expression of a proto-oncogene (proto-myb) in hemopoietic tissues of mice. Mol Cell Biol 4: 1206-1212, 1984

33. Schulz C, Gomez Perdiguero E, Chorro L, et al: A lineage of myeloid cells independent of Myb and hematopoietic stem cells. Science 336: 86-90, 2012

34. Shukla A and Yuspa SH: CLIC4 and Schnurri-2: A dynamic duo in TGF-beta signaling with broader implications in cellular homeostasis and disease. Nucleus 1: 144-149, 2010.

35. Shukla A, Malik M, Cataisson C, et al: TGF-beta signalling is regulated by Schnurri-2-dependent nuclear translocation of CLIC4 and consequent stabilization of phospho-Smad2 and 3. Nat Cell Biol 11: 777-784, 2009.

36. Staton TL, Lazarevic V, Jones DC, et al: Dampening of death pathways by schnurri-2 is essential for T-cell development. Nature 472: 105-109, 2011.

37. Nakayama $T$ and Kimura MY: Memory Th1/Th2 cell generation controlled by Schnurri-2. Adv Exp Med Biol 684: 1-10, 2010.

38. Kimura MY, Iwamura C, Suzuki A, et al: Schnurri-2 controls memory Th1 and Th2 cell numbers in vivo. J Immunol 178: 4926-4936, 2007.

39. Tu SW, Bugde A, Luby-Phelps K and Cobb MH: WNK1 is required for mitosis and abscission. Proc Natl Acad Sci USA 108: 1385-1390, 2011.

40. Jiang ZY, Zhou QL, Holik J, et al: Identification of WNK1 as a substrate of Akt/protein kinase $\mathrm{B}$ and a negative regulator of insulin-stimulated mitogenesis in 3T3-L1 cells. J Biol Chem 280: 21622-21628, 2005.

41. Sun X, Gao L, Yu RK and Zeng G: Down-regulation of WNK1 protein kinase in neural progenitor cells suppresses cell proliferation and migration. J Neurochem 99: 1114-1121, 2006.

42. Lee BH, Chen W, Stippec S and Cobb MH: Biological cross-talk between WNK1 and the transforming growth factor beta-Smad signaling pathway. J Biol Chem 282: 17985-17996, 2007.

43. Gottipati S, Rao NL and Fung-Leung WP: IRAK1: a critical signaling mediator of innate immunity. Cell Signal 20: 269-276, 2008.

44. An H, Hou J, Zhou J, et al: Phosphatase SHP-1 promotes TLRand RIG-I-activated production of type I interferon by inhibiting the kinase IRAK1. Nat Immunol 9: 542-550, 2008.

45. Jacob CO, Zhu J, Armstrong DL, et al: Identification of IRAK1 as a risk gene with critical role in the pathogenesis of systemic lupus erythematosus. Proc Natl Acad Sci USA 106: 6256-6261, 2009. 